\title{
Investigation of Part Feeding Problems in Manual Assembly Line
}

\author{
Nareerat Kumnuch ${ }^{1, a}$, Suksan Prombanpong ${ }^{1, b}$ \\ ${ }^{1}$ Department of Production Engineering, King Mongkut's University of Technology Thonburi (KMUTT), Bangkok, Thailand
}

\begin{abstract}
In a manual assembly line where different parts are consecutively assembled by workers along the conveyor line, line efficiency is dependent upon efficiency of part replenishment to the assembly line. If all parts can be served to the line on time, delay due to part interruption will not happen and jeopardize line efficiency. To avoid this adverse effect situation, a careful planning of feeding mechanism is a must and cannot overlook in a planning process. An optimum number of manpower, remaining buffer size, accurate feeding schedule, good logistic management, quick equipment maintenance and so on must be taken into account. Thus, this paper demonstrates a determination of optimum number of feeding worker, and feeding schedule based on different remaining buffer size. The equations formulated in this research and example of calculation will be delineated in details through a case study.
\end{abstract}

\section{Introduction}

A manual assembly line is a production line that consists of a sequence of workstations where assembly tasks are performed by human [1]. Factors attributed to a manual assembly line are high product demand, part identicalness, and task divisibility. The productivity of the assembly line mainly depends upon intermittent line stoppage due to the fact that new parts are not feeding in time. Therefore, feeding workers are of important to prevent the line stoppage. $\mathrm{Li}$ et al. approximated feeder line reliability in assembly systems [2]. It is common in many manufacturing plants to build subassemblies that feed the mainline. In modern manufacturing environment, more and more feeder lines or subassemblies are introduced to increase the flexibility and agility of the main manufacturing line [3,4,5]. A typical assembly system consists of one or more feeder lines which join a main line at an assembly station $[6,7,8]$. It can be noticed that most of research papers suggested building feeder lines to support main assembly station which assumes that the subassemblies feeder lines will continuously serve work parts in time.

However, a typical lengthy assembly line still utilizes a manual feeding of part to the assembly station and yet research in determining the optimal number of feeding workers has never been addressed although it is a chronic problem that reduces the productivity of the assembly lines nowadays. Some critical assembly lines even overuse feeding workers just to prevent line stoppage. Here is the first time that the optimum number of feeding workers can be determined by the formulated equations.

\section{Problem Scenario}

One of the manual assembly lines is investigated and it is consisted of a large number of assembly stations where parts are assembled to the base part and flows from left to right as shown in Fig. 1. The rectangular block represents a pallet where parts indicated by alphanumeric characters, are sorted in and positioned along the assembly line. There are a total of fourteen pallets (blocks) and once any of these pallets is empty, the new one will be replenished by a feeding worker. It can be imagined that if feeding workers are not enough or inefficient, the assembly line suffers low production rate. Thus, advance feeding depending upon buffer size should be planned.

In brief, problem related to feeding performance is an intermittent stop of the assembly line. Thus, the research issue is a determination of an optimal number of feeding worker, a proper pallet size and feeding schedule so that the assembly line will never suffer a cessation.

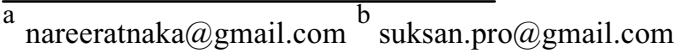




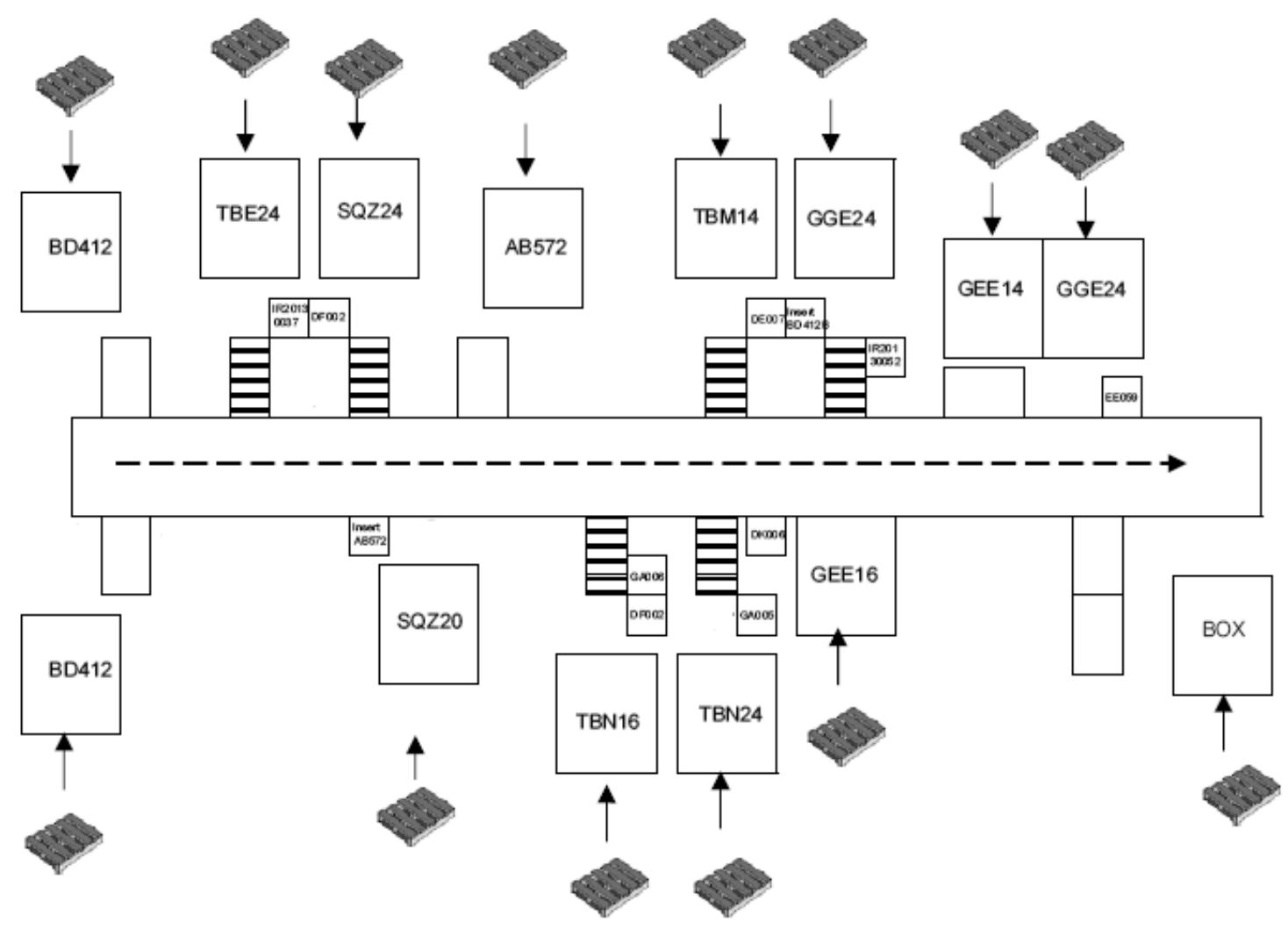

Figure 1. Layout of manual assembly line (a case study)

\section{Methodology}

Again, the research issue is a determination of an optimum number of feeding workers, mentioned in problem scenario section. Thus, feeding workload must be calculated. This can be done by collecting relevant data for calculation which is tabulated in Table 1.

Table 1 Initial relevant information

\begin{tabular}{|c|c|c|c|}
\hline $\begin{array}{l}\text { No. of } \\
\text { Stations }\end{array}$ & $\begin{array}{c}\text { Pallet size } \\
\text { (pcs.) }\end{array}$ & $\begin{array}{c}\text { Cycle time } \\
\text { (min) }\end{array}$ & $\begin{array}{c}\text { Feeding Time } \\
\text { (min /pallet) }\end{array}$ \\
\hline 14 & 300 & 1 & 2 \\
\hline
\end{tabular}

\section{Optimum Number of Feeding Workers}

This assembly line is consisted of 14 stations and each station equips with one pallet containing 300 parts. The cycle time of this line is $1 \mathrm{~min}$ and feeding operation time is 2 min per pallet. Note that in order to prevent line stop new pallet must be replenished before it is running out. Thus, each station must have a storage buffer for remaining parts which can place a few work-parts during replenishment. It can imagine that remaining buffer size also plays an important role to a number of feeding workers. If buffer stock is large, one worker can serve

more pallets in one time providing that the line must not be stop or wait for the new work-parts. In other words, the larger the remaining buffer stock is, the more pallets are. Therefore, the maximum number of feeding pallets can be determined from Eq.1

No. of feeding pallet $=$ Maximum integer

$\leq \frac{\text { Buffer } \times \text { Cycle time }}{\text { Feeding time }}$

Once the maximum number of pallets which one feeding workers can service, the optimum number of feeding workers can be determined from Eq.2

No. of workers $=$ Minimum integer

$\geq \frac{\text { no. of work stations }}{\text { no. of feeding pallets }}$

\section{Examples and Result}

Case 1: Assume that the remaining buffer size is 14 pieces and remaining data is the same as in Table 1. Thus, substitute the remaining buffer 14, cycle time 1 min and feeding time 2 min in Eq. 1

$$
\begin{aligned}
& \text { No. of feeding pallet = Maximum integer } \\
& \leq \frac{\text { Buffer } \times \text { Cycle time }}{\text { Feeding time }} \\
& \leq \frac{14 \times 1}{2}=7
\end{aligned}
$$


It means that one worker can feed up to 7 pallets before all the parts in the buffer storage are running out and make the assembly line stop. It is due to the fact that the number of workstation is equal to 14. Thus, substitute in Eq. 2

No. of workers $=$ Minimum integer

$\geq \frac{\text { no. of work stations }}{\text { no. of feeding pallets }}$

$\geq \frac{14}{7} \quad=2$

Thus, the optimum number of feeding workers is equal to 2. In other words, two feeding workers must be assigned to serve parts to the assembly line. The feeding schedule can be shown in Table 2. It should be noted that Time in Table 2 indicates the minute after the assembly line has been started. For example, after the assembly line has been worked for 16 minutes, the feeding worker 1 and 2 must start to replenish the first pallet. Each worker will spend two minutes to complete the work and then he/she can work on pallet $2,3, \ldots 7$ respectively. It can be imagined that once the remaining buffer of each station is finished, the assembly worker will start to use work-parts from the next pallet and it is considered as completion of a feeding round. Thus, the feeding worker will wait for another 16 minutes to start replenish the pallet for the next round. Typically, worker will use this free time to prepare the pallets for the next round or to work as utility worker; however, this worker must be back for duty within the designed schedule.

Table 2 Feeding schedule in the case of 14- remaining buffer size

\begin{tabular}{|c|l|l|}
\hline \multirow{2}{*}{ Time (minute at) } & \multicolumn{2}{|c|}{ Feeding worker } \\
\cline { 2 - 3 } & Worker 1 & Worker 2 \\
\hline 16 & BD412-1 & BD412-2 \\
\hline 18 & TBE24 & SQZ20 \\
\hline 20 & SQZ24 & AB572 \\
\hline 22 & TBM14 & TBN16 \\
\hline 24 & GEE24-1 & TBN24 \\
\hline 26 & GEE14 & GEE16 \\
\hline 28 & GEE24-2 & BOX \\
\hline
\end{tabular}

Case 2: This case is to demonstrate a calculation when the remaining buffer is 9 pieces and other data is the same as in Table 1. Thus, substitute the remaining buffer 9 pieces, cycle time $1 \mathrm{~min}$ and feeding time $2 \mathrm{~min}$ in Eq. 1
No. of feeding pallet $=$ Maximum integer

$\leq \frac{\text { Buffer } \times \text { Cycle time }}{\text { Feeding time }}$

$\leq \frac{9 \times 1}{2}$

$\leq 4.5$

Thus, the maximum integer which is less than or equal to 4.5 is 4 . Therefore, one worker can service up to 4 pallets in one round. Then, substitute number of pallet in Eq. 2

No. of workers $=$ Minimum integer

$\geq \frac{\text { no. of work stations }}{\text { Number of feeding pallets }}$

$\geq \frac{14}{4}$

$\geq 3.5 \approx 4$

Thus, the optimum number of worker which is the minimum integer greater than or equal 3.5 is 4 . The feeding schedule in the case of 9-remaining buffer size is shown in Table 3.

Table 3 Feeding schedule in the case of 9- remaining buffer size

\begin{tabular}{|l|l|l|l|l|}
\hline \multirow{2}{*}{$\begin{array}{c}\text { Time } \\
\left(\begin{array}{c}\text { minute } \\
\text { at }\end{array}\right.\end{array}$} & \multicolumn{4}{|c|}{ Feeding worker } \\
\cline { 2 - 5 } & Worker 1 & Worker 2 & Worker 3 & Worker 4 \\
\hline 22 & BD412-1 & BD412-2 & TBE24 & SQZ24 \\
\hline 24 & SQZ20 & TBN16 & AB572 & TBN24 \\
\hline 26 & TBM14 & GEE16 & GEE24-1 & \\
\hline 28 & GEE14 & GEE24-2 & BOX & \\
\hline
\end{tabular}

Again, all four workers will start replenishing the pallets after the assembly line has been started for 22 minutes and continue working to serve up to four pallets to complete the first round except worker 4 will feed only up to 3 pallets. In this manner, the feeding schedule can be established and the feeders can attend their work on time.

\section{Conclusion and Discussion}

This paper presents the formulated equations to determine an optimal number of feeding workers for a manual assembly line. The number of workers mainly depends on the cycle rate, remaining buffer size, feeding operation time. It is found that with an increasing of remaining buffer size, a number of required workers will be lesser since one worker can serve more pallets to other stations. The example of two different sizes of remaining buffer capacity is utilized. The pallet size of each workstation in this paper assumes equal; therefore, an assembly line with various sizes of pallet and buffer sizes should be further investigated. 


\section{References}

1. M.P. Groover: Automation Production Systems and Computer-Integrated Manufacturing, $3^{\text {rd }}$ Edition, Prentice Hall Press Publishers, USA. (2008)

2. J. Li, J.M. Alden and J.R Rabaey: Approximating feeder line reliability statistics in assembly systems with partial data collection, Computers and Industrial Engineering, Vol. 48 (2005), pp. 181-203

3. J. Li, J.M. Alden and J.R Rabaey: Approximating feeder line reliability statistics in assembly systems with partial data collections, Technical Report R \& D-9391. Warren, General Motors Research and Development center (2002)

4. T. Altiok: Performance analysis of manufacturing systems, Springe-Verlag New York Berlin Heidellberg Publishes (1997)
5. S.Y. Chiang, C.T Kuo, J.T. Lim and S.M. Meerkov: Improvability theory of assembly systems I: problem formulation and performance evaluation, Mathematical Problems in Engineering, Vol. 6 (2000), pp. 321-357

6. S.Y. Chiang, C.T. Kuo, J.T. Lim and S.M. Meerkov: Improvability theory of assembly systems II: improvability indicators and case study, Mathematical Problems in Engineering, Vol. 6 (2000), pp. 359-393

7. J.T. Simon and W.J. Hopp. Availability and average inventory of balanced assembly-like flow systems. IIE Transactions, Vol 23(1991), pp. 161-168.

8. S.B.Gershwin and M.H. Burman: A decomposition method for analyzing in homogeneous assembly/disassembly systems, Annals of Operations Research, Vol. 93 (2000), pp. 91-115 